beklagte Hans-Jochen Vogel fehlende Plebiszite im Bund (S. 9 f.), doch weder er noch die SPD stritten für die Volksabstimmung über die Verfassung des vereinten Deutschland in Art. 146 GG a. F. Das Buch dürfte über das Plakative hinaus kaum Wirkung entfalten. Weder gibt es einen Diskurs mit den Gegnern von und Zweiflern an Plebisziten, noch geht es auf die sich ausdifferenzierende Gesellschaft ein; sie halten mangels Homogenität die Parteien zusammen. So schadet „Mehr Demokratie“ eher dem notwendigen Mehr an Demokratie.

Erich Röper

\title{
Schabowskis Lektion: schonungslose Kritik am real existierenden Sozialismus
}

Günter Schabowski im Gespräch mit Frank Sieren, Wir haben fast alles falsch gemacht. Die letzten Tage der DDR, Econ Verlag, Berlin 2009, 281 Seiten, € 19,90.

Günter Schabowski, im Jahre 200980 Jahre alt geworden, ist ein Solitär - der einzige führende SED-Politiker, der die diktatorische Vergangenheit und seine Rolle dabei nicht beschönigt. Ende 1999 zu drei Jahren Haft wegen Totschlags vom Landgericht Berlin verurteilt, kam er nach einem Jahr durch Begnadigung des Regierenden Berliner Bürgermeisters frei. Seine Erklärungen vor dem Berliner Landgericht sind im Anhang des hier anzuzeigenden Buches abgedruckt. „Das System, dessen politischer Klasse ich seit 1981 als Kandidat und seit 1984 als Mitglied des Politbüros der SED angehört habe, hat vor dem Leben, vor der Wirklichkeit versagt.“ (S. 256) Obwohl sich moralisch schuldig fühlend, leugnete er eine juristische Verantwortung allein aufgrund seiner Mitgliedschaft im Politbüro. Er sei, da kein Mitglied des Nationalen Verteidigungsrates, niemals an einer Entscheidung zum Töten von Menschen an der Grenze beteiligt gewesen. Schuld und Schmach nahm Schabowski gleichwohl auf sich. So wandte er sich gegen das in den Reihen der PDS benutzte Wort von der „Siegerjustiz“.

Bereits 1990 hatte Frank Sieren einen Interviewband mit Günter Schabowski herausgegeben. ${ }^{1}$ Fast 20 Jahre später folgt eine Art Fortsetzung. In zwölf Kapiteln geht es um - natürlich - die Öffnung der Mauer, das Sich-Einleben in die Demokratie, die Karriere in der DDR, die Entstehung des „Arbeiter- und Bauern-Staates“, die Ideologie der kommunistischen Verschwörung, die Staatssicherheit, die Bedeutung des 17. Juni 1953, das Verhältnis Moskau-(Ost-)Berlin, die deutsch-deutschen Beziehungen, die „profitfreie Planwirtschaft“, die Staatsmedien, die Verwandlung der SED zur PDS/Linke. Der Interviewte, ein brillanter Formulierer, nimmt kein Blatt vor den Mund. Die Fragen Sierens sind mehr Stichworte, basieren weniger auf kritischem Nachhaken. Insofern hat es Schabowski leicht, gerät niemals in die Defensive. Gleichwohl wirkt seine Position nicht nur glaubwürdig, sondern auch überzeugend. Allerdings erfährt der Leser, der Schabowskis Positionen kennt, nicht sonderlich viel Neues. Jetzt setzt der Autor die Akzente mit Blick auf seine Rolle bei der

1 Vgl. Günter Schabowski, Das Politbüro. Ende eines Mythos. Eine Befragung, herausgegeben von Frank Sieren / Ludwig Koehne, Hamburg 1990. 
legendären Pressekonferenz am 9. November 1989 etwas anders. Die Rolle der Medien kommt immer noch zu knapp weg. ${ }^{2}$

Betont wird stärker sein aktives, zielgerichtetes Handeln. Aber war er nicht selber von den atemberaubenden Folgen der Pressekonferenz überrascht? Wenn die Bekanntgabe der Reisefreiheit und des Rechts auf ständige Ausreise ein „Knaller“ (Egon Krenz) sein sollte, dann ist die folgende Feststellung schwerlich nachvollziehbar: „Ich habe diese Formulierung in schnellem Tempo vorgelesen, weil ich vor der Öffentlichkeit nicht auch noch betonen wollte, dass das ein Schritt aus der Bedrängnis war." (S. 31) Völlig ging bei dieser Pressekonferenz die sensationelle Ankündigung unter, freie Wahlen durchzuführen.

Im Vergleich zu dem Interviewband und dem 1991 publizierten Buch „Der Absturz“3 rechnet Schabowski nicht nur mit der Realität der SED-Diktatur ab, sondern auch mit deren weltanschaulicher Grundlage. Die Praxis sei eine konsequente Folge der Theorie. Schabowski zeigt sich in der Kenntnis der marxistischen Ideologie und der Kritik an ihr (zum Beispiel von Raymond Aron oder Arthur Koestler) überaus beschlagen. Wäre dem Leser nicht mehr gedient gewesen, wenn Schabowski stärker zu seinen Karriereschritten befragt worden wäre, als dass er seine theoretisch fundierte Kritik an den Klassikern ausbreitet?

Schabowskis an sich konsistentes Gedankengebäude ist in einem Punkt nicht widerspruchsfrei. So heißt es: „Das Aus der DDR ist das Resultat einer Reihe von Faktoren: Dazu gehörten der Mangel an Freizügigkeit, der durch die ungarische Grenzöffnung beklemmend offenbar wurde, die erstickende Allgegenwart der Stasi, das Fehlen echter Demokratie, Versorgungsprobleme, der täglich verordnete Realitätsentzug in den Medien ebenso wie unsere Nachholverbesserungen wie etwa die Maueröffnung, der zögerliche Rückzug der SED aus der selbstverhängten Führungsrolle oder der klägliche Zerfall der scheinbar monolithischen Partei.“ (S. 133 f.) Tatsächlich war weder „der Mangel an Freizügigkeit“ noch „das Fehlen echter Demokratie“ eine Ursache für den Untergang der diktatorischen DDR, denn, wie Schabowski an anderer Stelle richtig sagt: „Kommunistische Staaten waren und sind nicht reformfähig.“ (S. 196) Insofern hätten „Freizügigkeit“ und „Demokratie“ das System eher zum Einsturz gebracht.

Im Kern richtig wird die Position Gorbatschows erfasst, auch wenn Schabowskis Interpretation zumal in Deutschland nicht sonderlich populär ist. Er spricht von einer „naive(n) Gorbi-Manie“ (S. 174), ohne jedoch die historische Rolle Gorbatschows zu schmälern. Dessen Perestroika „erwies sich als eine totale Illusion. Seine Reformversuche lieferten den nützlichen Beweis für die Unreformierbarkeit des Systems“ (S. 170). Besonders schlecht kommen Gregor Gysi, Hans Modrow und Markus Wolf weg, die Gorbatschow noch bis in den Februar 1990 einzureden versucht hätten, eine Eigenständigkeit der DDR sei nicht ohne realistische Chance.

Mit der PDS, der Linkspartei und der Linken geht Schabowski hart ins Gericht. Diese Partei hätte verboten werden müssen. Der Autor kritisiert ihre „Robin-Hood-Attitüde“ (S. 240); sie sei wesentlich für die Missstände verantwortlich gewesen, gegen die sie später protestiert habe. Schabowski spricht sarkastisch von den "Beton-Marxisten“, die die „Gummi-Marxisten“ brauchen; und die „Polit-Elastokraten“ benötigten die „versteinerte

2 Vgl. Hans-Hermann Hertle, Chronik des Mauerfalls. Die dramatischen Ereignisse um den 9. November 1989, Berlin 2009, S. 278 - 291 (Nachwort zur 11. Auflage).

3 Vgl. Günter Schabowski, Der Absturz, Berlin 1991. 
Menge“. „Die Linke/PDS ist also eine Zwangssymbiose aus Vorturnern und Steif-Riegen.“ (S. 246)

Das Buch gibt einen authentischen Einblick in die Vorstellungswelt eines einstmals führenden Kommunisten, der schon lange über seinen Schatten gesprungen ist. Die Kritik am realen Sozialismus ist fundamentaler, die Verteidigung der parlamentarischen Demokratie prinzipieller Natur. Schabowski, der seine bittere Lektion gelernt hat, erteilt nun Unbelehrbaren seinerseits eine Lektion, keineswegs in belehrendem Duktus.

Eckhard Jesse

\section{Rechtsextremismus: Neue Forschungsbeiträge mit vielen Details und aktueller Problemsicht}

Braunthal, Gerard: Right-Wing Extremism in Contemporary Germany, Palgrave Macmillan, Houndmills / Basingstoke 2009, 280 Seiten, € 64,-.

Weckenbrock, Christoph: Die streitbare Demokratie auf dem Prüfstand. Die neue NPD als Herausforderung, Bouvier, Bonn 2009, € 25,-.

Christoph Weckenbrock und Gerard Braunthal nähern sich auf unterschiedliche Weise einem ähnlichen Forschungsgegenstand. Beide Arbeiten beleuchten den Erfolg der extremen Rechten in Deutschland, seine gesellschaftlichen Hintergründe sowie die Optionen einer freiheitlich-demokratischen Gesellschaftsordnung, der freiheits- und demokratiefeindlichen Idee des Rechtsextremismus wirksam zu begegnen. Während die in ihrer Vielzahl an Informationen beeindruckende Untersuchung von Gerard Braunthal den Rechtsextremismus in Deutschland in all seinen Facetten beleuchtet, stellt die Arbeit von Christoph Weckenbrock die streitbare Demokratie in den Vordergrund. Analysiert werden vor dem Hintergrund der jüngsten Erfolge der NPD die Konzeption der streitbaren Demokratie, ihre Interpretation im politik- und rechtswissenschaftlichen Diskurs sowie die Auswirkungen der Erfolge der NPD auf dieses Demokratieschutzprinzip.

Braunthal charakterisiert den parteipolitischen Rechtsextremismus auf der Grundlage typischer Einstellungs- und Verhaltensmuster. Hierzu werden Merkmale wie Nationalismus, Rassismus, Xenophobie, Antisemitismus sowie das soziale beziehungsweise psychosoziale Geschlecht gezählt. Untersucht wird in der Folge die historische Entwicklung des parteipolitischen Rechtsextremismus in Deutschland. Aufgrund des hierzu eher übersichtlichen Forschungsstandes ist der Abschnitt über rechtsextreme Tendenzen in der DDR hervorzuheben. Der von der Partei- und Staatsführung der DDR propagierte Antifaschismus und das beharrliche Verneinen rechtsextremer Tendenzen in der DDR halten einer empirischen Untersuchung nicht stand. Allerdings hat sich der Rechtsextremismus der DDR nicht in Parteien organisiert (S. 36 ff.). Vielmehr gab es mehr oder weniger lose organisierte Gruppierungen, deren zumeist jugendliche Mitglieder dem sozialistischen System insbesondere in den späten 1980er Jahren kritisch gegenüberstanden (S. 41). Insbesondere in den letzten Jahren des DDR-Regimes kam es zu einer Radikalisierung dieser Gruppen, die sich in gewaltsamen Übergriffen und der Schändung jüdischer Friedhöfe und Gedenkstät- 